\title{
Complete Genome Sequence and Biodegradation Characteristics of Benzoic Acid-Degrading Bacterium Pseudomonas sp. SCB32
}

\author{
Wei Xiang $\mathbb{D}^{1}{ }^{1}$ Xiaolan Wei $\left(\mathbb{D},{ }^{1}\right.$ Hui Tang, ${ }^{2}$ Liangbo Li $\mathbb{D}^{1},{ }^{1}$ and Rongshao Huang $\mathbb{D}^{1}$ \\ ${ }^{1}$ Department of Agronomy, Agricultural College of Guangxi University, Nanning 530004, China \\ ${ }^{2}$ Guangxi Institute of Botany, Chinese Academy of Sciences, Guilin 541006, China
}

Correspondence should be addressed to Liangbo Li; 1lb100@126.com and Rongshao Huang; hrshao802@163.com

Received 23 December 2019; Accepted 17 April 2020; Published 3 July 2020

Academic Editor: Shin-ichi Yokota

Copyright (C) 2020 Wei Xiang et al. This is an open access article distributed under the Creative Commons Attribution License, which permits unrestricted use, distribution, and reproduction in any medium, provided the original work is properly cited.

\begin{abstract}
Allelochemicals are metabolites produced by living organisms that have a detrimental effect on other species when released into the environment. These chemicals play critical roles in the problems associated with crop replanting. Benzoic acid is a representative allelochemical found in root exudates and rhizosphere soil of crops and inhibits crop growth. The bioremediation of allelochemicals by microorganisms is an efficient decontamination process. In this research, a bacterial strain capable of degrading benzoic acid as the sole carbon source was isolated. The genome of the strain was sequenced, and biodegradation characteristics and metabolic mechanisms were examined. Strain SCB32 was identified as Pseudomonas sp. based on 16S rRNA gene analysis coupled with physiological and biochemical analyses. The degradation rate of $800 \mathrm{mg} \mathrm{L}^{-1}$ benzoic acid by strain SCB32 was greater than $97.0 \%$ in $24 \mathrm{~h}$. The complete genome of strain SCB32 was $6.3 \mathrm{Mbp}$ with a GC content of $64.6 \%$ and 5960 coding genes. Potential benzoic acid degradation genes were found by comparison to the KEGG database. Some key intermediate metabolites of benzoic acid, such as catechol, were detected by gas chromatography-mass spectrometry. The biodegradation pathway of benzoic acid, the ortho pathway, is proposed for strain SCB32 based on combined data from genome annotation and mass spectrometry. Moreover, the benzoic acid degradation products from strain SCB32 were essentially nontoxic to lettuce seedlings, while seeds in the benzoic acid-treated group showed significant inhibition of germination. This indicates a possible application of strain SCB32 in the bioremediation of benzoic acid contamination in agricultural environments.
\end{abstract}

\section{Introduction}

Aromatic compounds, widely found in the environment as components of plant materials and as pollutants of anthropogenic sources, are usually toxic to cellular systems and must be removed [1]. Benzoic acid is the simplest aromatic acid and a metabolic intermediate for many aromatic compounds [2-4]. Ecosystem pollution resulting from an increase in benzoic acid or sodium benzoate in soil and water poses a hazard to humans and other organisms in the ecosystem. Benzoic acid is commonly used as a pharmaceutical or as an industrial preservative and can also be used in synthetic fibers, coatings, resins, tobacco, and rubber industries. The European Commission determined the toxicity level of benzoic acid in 2005, and the toxicology and adverse effects of ben- zoic acid have been widely reviewed [5]. The wide application of benzoic acid has resulted in it becoming a common environmental pollutant. In agriculture, benzoic acid also demands attention. Autotoxicity, a common phenomenon in natural and agroecosystems, occurs when a living or decomposing plant releases toxins into the surrounding environment to inhibit the growth and development of the same species [6]. In crop root exudates or rhizosphere soil, benzoic acid and its derivatives accumulate over years of cultivation. Benzoic acid is considered to be the predominant cause of crop autotoxicity [7-9], leading to nutrient imbalance and microbial dysfunction in the soil, which cause continuous cropping obstacles $[10,11]$.

Traditionally, crop rotation can solve the problems associated with continuous cropping. However, this method is 
inefficient, so there is an urgent need to develop alternative sustainable techniques. Microbial degradation of benzoic acid or other allelochemicals in the soil determines plant autotoxicity activity $[12,13]$. Panax notoginseng (Sanqi) is a valuable medicinal material in China with serious continuous cropping obstacles $[14,15]$. Our previous studies have shown that there are more organic acids in rhizosphere soil than in uncultivated soil. Benzoic acid has been found in the root exudates of Sanqi, where it significantly inhibited growth [16]. During our investigations, we found some wild cultivars of Sanqi that have been continuously cultivated for 8-10 years in the same field, and these cultivars grow healthily. Usually, after harvesting Sanqi, it is impossible to continue planting in the same field due to the problems of continuous cropping [15]. We determined that the differences between cultivars and continuous cultivation of Sanqi may be related to rhizosphere microorganisms. Such microorganisms should be highly tolerant of allelochemicals and could be used for biodegradation. Researchers have identified a variety of microorganisms that can degrade benzoic acid, such as Pseudomonas, Syntrophus, and Streptomyces [17, 18]. Although biodegradation is a highly efficient technique, numerous factors affect degradation efficiency, including $\mathrm{pH}$, temperature, and oxygen limitations [19]. In terms of the mechanism of degradation, benzoate biodegradation by Pseudomonas has been reported to occur via the ortho cleavage pathway [20] or by involving both the meta and ortho pathways depending on the concentration of benzoic acid [21]. Different key enzymes for various metabolic pathways of benzoic acid, such as 1,2-dioxygenase and 2,3-dioxygenase, have been found in bacteria $[22,23]$. Since the sequence of the first bacterial genome (Haemophilus influenzae $\mathrm{Rd}$ ) was completed in 1995 [24], the quantity of microbial genomic data has exploded [25]. In biodegradation studies, complete genome sequences can be used to rapidly interpret strain characteristics and predict degradation mechanisms [26, 27].

While some benzoic acid-degrading bacteria have been discovered, the large number of microbial resources warrants further exploration, and there is merit in studying the application of such microbes in bioremediation. In this study, the benzoic acid-degrading strain Pseudomonas sp. SCB32 was isolated from Sanqi continuous cropping soils. The effects of substrate concentration, $\mathrm{pH}$, and temperature on the biodegradation of benzoic acid by strain SCB32 were explored. The complete genome sequence of the strain SCB32 was determined, and degradation products of the strain were analyzed by gas chromatography-mass spectrometry (GCMS) to determine the intermediate metabolites of benzoic acid. A possible degradation pathway was deduced following the combined analysis of genome annotation and MS data. Moreover, the toxicity of degradation products of benzoic acid was measured to investigate potential applications of the strain. Isolating and identifying an efficient benzoic acid-degrading bacterial strain and exploring the mechanisms of degradation are of particular significance for the agricultural environment, as it potentially provides a new way to alleviate the problems associated with continuous cropping.

\section{Materials and Methods}

2.1. Chemicals and Medium. Luria-Bertani medium (LB, $\mathrm{pH} 7.0$ ) contained peptone $10.0 \mathrm{gL}^{-1}$, yeast extract $5.0 \mathrm{gL}^{-1}$, and $\mathrm{NaCl} 10.0 \mathrm{gL}^{-1}$. A mineral salt medium (MSM, pH 7.0) contained (NH4) ${ }_{2} \mathrm{SO}_{4} 1.0 \mathrm{gL}^{-1}, \mathrm{KH}_{2} \mathrm{PO}_{4} 0.5 \mathrm{gL}^{-1}, \mathrm{~K}_{2} \mathrm{HPO}_{4}$ $1.5 \mathrm{gL}^{-1}, \mathrm{NaCl} 1.0 \mathrm{gL}^{-1}$, and $\mathrm{MgSO}_{4} \cdot 7 \mathrm{H}_{2} \mathrm{O} 0.1 \mathrm{gL}^{-1}$. Solid medium plates were prepared by adding $18 \sim 20 \mathrm{~g} \mathrm{~L}^{-1}$ agar into the abovementioned liquid media. All media were sterilized by autoclaving at $121^{\circ} \mathrm{C}$ for $30 \mathrm{~min}$. Benzoic acid $(99.5 \%$ purity) was purchased from Sinopharm Chemical Reagent Beijing Co., Ltd., China. All other chemical reagents were of analytical grade. Ultrapure water $\left(18.2 \mathrm{M} \Omega \mathrm{cm}^{-1}\right)$ was prepared with a YPK-11 water purification system (Youyue, Chengdu, China).

2.2. Enrichment and Isolation of Benzoic Acid-Degrading Bacteria. Rhizosphere soil of Panax notoginseng was sampled from Baise City $\left(23^{\circ} 34^{\prime} 11^{\prime \prime} \mathrm{N}, 105^{\circ} 55^{\prime} 56^{\prime \prime} \mathrm{E}\right)$, Guangxi Province, China. Five grams of moist soil sample was added to Erlenmeyer flasks containing $100 \mathrm{~mL}$ MSM supplemented with $50 \mathrm{mg} \mathrm{L}^{-1}$ benzoic acid. Cultures were incubated at $30^{\circ} \mathrm{C}$ and $180 \mathrm{rpm}$ for 7 days for enrichment. Thereafter, $10 \mathrm{~mL}$ of culture was sampled every 7 days and transferred to fresh MSM, in which the concentration of benzoic acid was gradually increased to 100,150 , and $200 \mathrm{mg} \mathrm{L}^{-1}$. Bacterial strains that degraded benzoic acid in the culture were isolated and purified following the procedure described by Buermans and den Dunnen [26]. All media, pipette tips, and Erlenmeyer flasks were sterilized by autoclaving at $121^{\circ} \mathrm{C}$ for $20 \mathrm{~min}$ before use.

2.3. Evaluation of Ability of Isolated Strains to Degrade Benzoic Acid. A single colony from an agar plate was inoculated into liquid MSM containing $200 \mathrm{mg} \mathrm{L}^{-1}$ benzoic acid and was incubated at $30^{\circ} \mathrm{C}$ and $180 \mathrm{rpm}$ to logarithmic phase. Bacteria were collected and washed with $0.05 \mathrm{M}$ PBS ( $\mathrm{pH} 7.0$ ), then resuspended in the same concentration of PBS to an $\mathrm{OD}_{600}$ of 1.0. Five milliliters of this bacterial culture was inoculated into $100 \mathrm{~mL} \mathrm{MSM} \mathrm{containing} 200 \mathrm{mg} \mathrm{L}^{-1}$ of benzoic acid. After incubation for $48 \mathrm{~h}$ at $30^{\circ} \mathrm{C}$ and $180 \mathrm{rpm}$, microbial growth was observed and the benzoic acid residue was quickly analyzed using a UV-1800 spectrophotometer (Shimadzu, Japan); the analysis method was performed as described by Mahboubifar et al. [28]. All experiments were performed three times.

2.4. Identification of Strain SCB32. Morphological characteristics were determined using an optical microscope (Nikon, Eclipse E100, Japan) after incubation for $24 \mathrm{~h}$ at $30^{\circ} \mathrm{C}$. Gram staining was performed according to the method described by Claus, and physiological and biochemical identification was accomplished using the VITEK GN test. Total DNA of strain SCB32 was extracted using a Bacterial Genomic DNA Extraction Kit (Sangon, China), and the 16S rRNA gene was amplified from genomic DNA using the universal primers $27 \mathrm{~F}$ and $1492 \mathrm{R}$ [29]. PCR products were purified and sequenced by Sangon Biotech (Shanghai, China). The resulting 16S rRNA gene sequences were submitted to GenBank and BLAST search by the EzBioCloud identification 
service [30], and average nucleotide identity (ANI) was determined through the TrueBac ID system [31]. The closest reference sequences were then obtained from the GenBank database, and a phylogenetic tree was generated using MEGA 6.1 software with a neighbor-joining algorithm (bootstrap analysis was performed with 1000 replicates).

2.5. Biodegradation Characterization of Strain SCB32. The effects of environmental factors on benzoic acid degradation by strain SCB32 were evaluated in a series of batch experiments performed in $500 \mathrm{~mL}$ Erlenmeyer flasks. The initial series determined the concentration range, optimum $\mathrm{pH}$, and temperature for benzoic acid degradation by selected strains. Single-factor optimization tests were applied for conditions including concentration ranges $(200,400,600,800$, 1000 , and $\left.1200 \mathrm{mg} \mathrm{L}^{-1}\right), \mathrm{pH}(5.0,6.0,7.0,8.0$, and 9.0), and temperature $\left(20,25,30,35\right.$, and $\left.40^{\circ} \mathrm{C}\right)$; in the $\mathrm{pH}$ and temperature tests, the initial concentration of benzoic acid was maintained at $800 \mathrm{mg} \mathrm{L}^{-1}$. The second series measured the degradation efficiency and growth of strain SCB32 under optimized conditions ( $800 \mathrm{mgL}^{-1}, \mathrm{pH} 7.0$, and $30^{\circ} \mathrm{C}$ ). The residual concentration and cell density of strain SCB32 were measured every $2 \mathrm{~h}$, and concurrently, another sample was stored at $-80^{\circ} \mathrm{C}$ for analysis of degradation pathways. For all experiments, the preparation method of the test bacterial solution was the same as above, and a culture medium without bacteria was used as a control.

2.6. Genome Sequencing and Bioinformatics Analysis. Genomic DNA was extracted using a QIAamp kit (Qiagen, Germany). The quantity of DNA was measured using a NanoDrop 2000 spectrophotometer (Thermo Scientific, USA). The genome of strain SCB32 was sequenced by Single Molecule, Real-Time (SMRT) technology (PacBio) at Novogene (Beijing, China). Low-quality reads were removed by SMRT Link v5.0.1, and the filtered reads were assembled to generate one contig without gaps. The genome was annotated through the databases GO (Gene Ontology) [32], KEGG (Kyoto Encyclopedia of Genes and Genomes) [33], COG (Clusters of Orthologous Groups) [34], NR (NonRedundant Protein Database databases) [35], and SwissProt protein database [36].

2.7. GC-MS Analyses of Benzoic Acid Metabolites. Cocultures of strain SCB32 were grown in $500 \mathrm{~mL}$ basal medium with $800 \mathrm{mg} \mathrm{L}^{-1}$ benzoic acid to detect metabolites of benzoate metabolism. Samples $(50 \mathrm{~mL})$ were withdrawn from the cultures at various times and centrifuged at $9500 \mathrm{rpm}$ and $4^{\circ} \mathrm{C}$ for $5 \mathrm{~min}$. The resulting supernatant was acidified with $200 \mu \mathrm{L} 12 \mathrm{M} \mathrm{HCL}$ and then extracted three times with $25 \mathrm{~mL}$ ethyl acetate [21]. Ethyl acetate extracts were dried under nitrogen gas and then derivatized with N,O-bis(trimethylsilyl)trifluoroacetamide (TMS). One microliter of the derivatized sample was injected into a GC-MS system (Varian 300-MS, USA). The GC conditions were as follows: carrier gas, helium; splitless mode; temperature programming, $50^{\circ} \mathrm{C}(5 \mathrm{~min}), 50-140^{\circ} \mathrm{C}\left(25^{\circ} \mathrm{C} \mathrm{min}^{-1}\right)$, and $140-250^{\circ} \mathrm{C}$ $\left(5^{\circ} \mathrm{C} \mathrm{min}^{-1}\right)$. Separation was achieved on a VF-5ms column
$(30 \mathrm{~mm} \times 0.25 \mathrm{~mm} \times 0.25 \mu \mathrm{m})$. Mass spectra were obtained at an electron impact (EI) of $70 \mathrm{eV}$.

2.8. Bioassay of Benzoic Acid Degradation Products. Seeds of lettuce (Lactuca sativa L.) cultivar "916" (Guangxi, China) were used for bioassay of benzoic acid degradation products. Lettuce seeds were sterilized with sodium hypochlorite and placed in a $9 \mathrm{~cm}$ diameter Petri dish containing two layers of sterile filter paper. The filter paper was pretreated with $10 \mathrm{~mL}$ benzoic acid degradation products (strain SCB32 was inoculated into liquid MSM containing $800 \mathrm{mg} \mathrm{L}^{-1}$ benzoic acid for $48 \mathrm{~h}$ ), MSM with $800 \mathrm{mg} \mathrm{L}^{-1}$ benzoic acid, or MSM alone. Thirty seeds were placed in each dish, and each treatment was repeated three times. The seeds were placed in an incubator at $25^{\circ} \mathrm{C}$ with a $12 \mathrm{~h}$ light $/ 12 \mathrm{~h}$ dark photoperiod and kept moist during incubation. After 7 days, the germination rate and fresh weight were measured.

2.9. Statistical Analyses. All analyses were performed using SPSS v24.0 software. All datasets were subjected to an analysis of variance (ANOVA) and expressed as mean \pm SE. Statistical significance of differences $(p<0.05)$ was assessed by Duncan's multiple range test.

\section{Results}

3.1. Isolation and Identification of Benzoic Acid-Degrading Strains. After 5 weeks of enrichment, domestication, and separation, four strains of benzoic acid-degrading bacteria were obtained from the soil samples. All four strains could grow in MSM with benzoic acid as the sole carbon source. The isolates exhibited variable degradation capacity (32.90-84.34\%) with $200 \mathrm{mgL}^{-1}$ benzoic acid in liquid MSM (data not shown). Strain SCB32 showed the greatest degradation ability and was therefore selected for further study. Cells of strain SCB32 were Gram stain-negative, rod-shaped, and 0.3-0.7 $\mu$ $\mathrm{m} \times 0.9-2.1 \mu \mathrm{m}$ and were arranged singly or in pairs. Colonies of the isolate were light yellow, round, convex, and moist with bacterial opacity when cultured on LB agar for $24 \mathrm{~h}$ at $30^{\circ} \mathrm{C}$ (Figure 1). Based on 16S rRNA gene sequence comparison and phylogenetic analysis (Figure 2), strain SCB32 had 99.66\% similarity to Pseudomonas nitritireducens WZBFD3$5 \mathrm{~A} 2^{\mathrm{T}}$. Additionally, ANI analysis showed that the strain was more than $80 \%$ similar to the member of the genus Pseudomonas. On the basis of these results, the isolate was designated Pseudomonas sp. SCB32. VITEK GN test results and ANI data are shown in Supplementary Tables S1 and S2.

3.2. Characterization of Benzoic Acid-Degrading Strain SCB32. Degradation of benzoic acid by strain SCB32 at different initial concentrations, temperatures, and $\mathrm{pH}$ is shown in Figure 3. After incubation for $48 \mathrm{~h}$, the degradation rate of benzoic acid at a concentration of $800 \mathrm{mg} \mathrm{L}^{-1}$ was greater than $97.0 \%$. However, when the concentration of benzoic acid was further increased to 1000 or $1200 \mathrm{mg} \mathrm{L}^{-1}$, the degradation rate dropped significantly $(p<0.05)$ to $31.1 \%$ (Figure $3(\mathrm{a})$ ). The effects of temperature and $\mathrm{pH}$ were therefore explored using $800 \mathrm{mg} \mathrm{L}^{-1}$ benzoic acid. Strain SCB32 could effectively degrade benzoic acid at pH 5.0 to 9.0 (Figure 3(b)), with the degradation rate greater than $80.0 \%$ in all cases. The rate of 


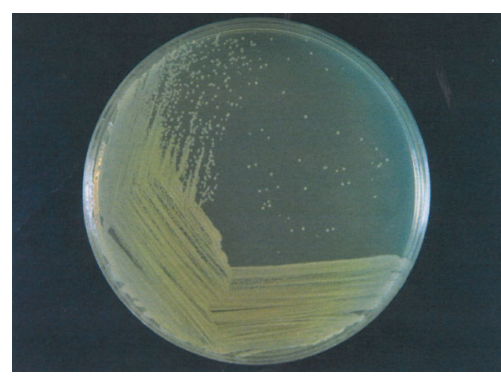

(a)

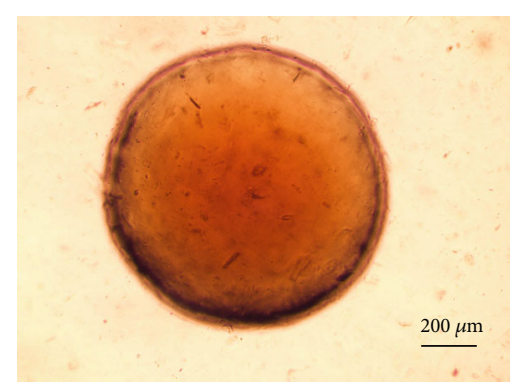

(b)

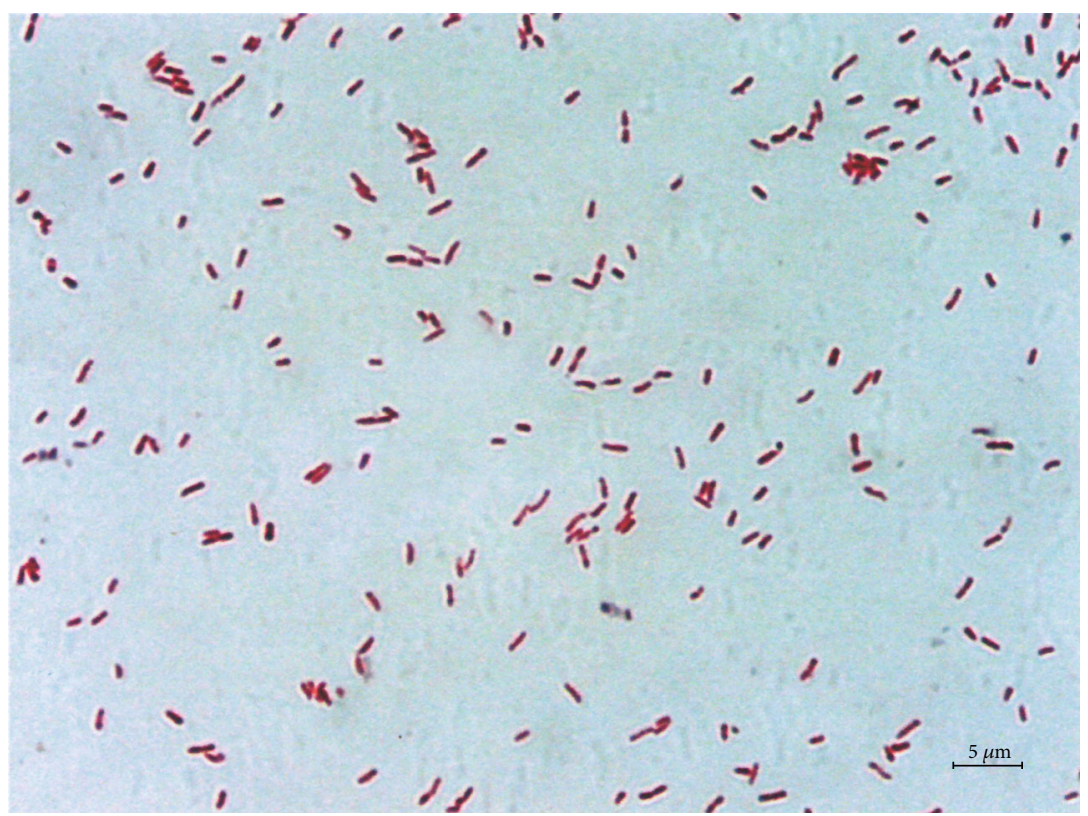

(c)

FIgURE 1: Morphological features of strain SCB32. Colony morphology (a), colony morphology (b), and Gram staining (c).

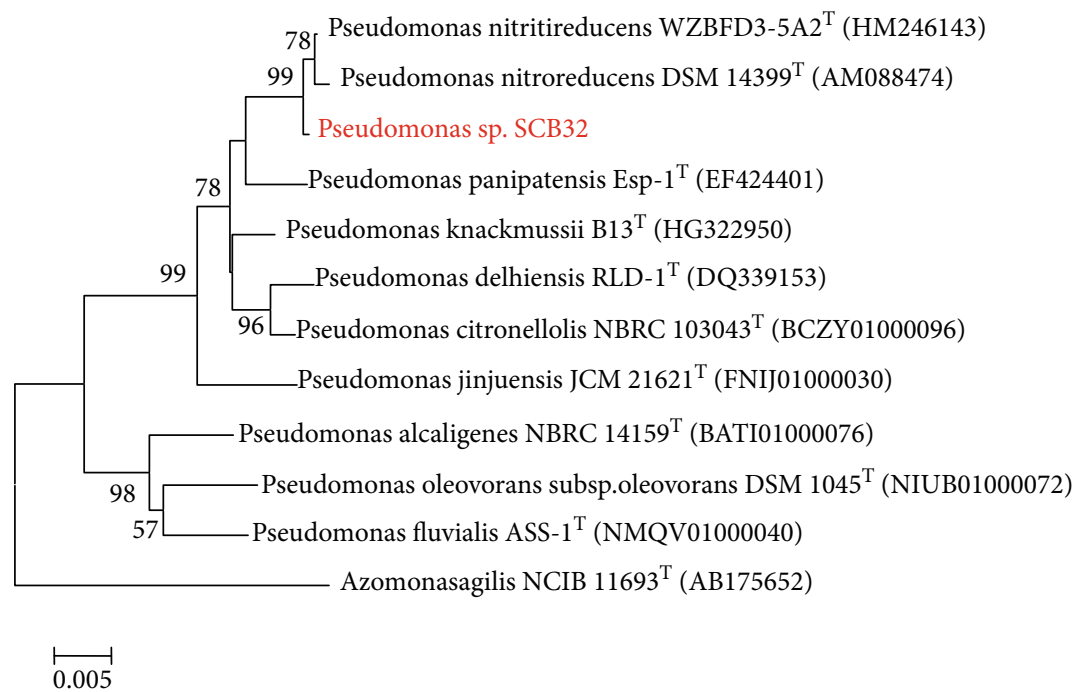

FIGURE 2: Phylogenetic tree based on $16 \mathrm{~S}$ rRNA gene sequences showing the relationship between the members of the genus Pseudomonas. Bootstrap values (percentages) are based on 1000 replicates and are shown for branches with more than 50\% bootstrap support. Superscript "T" indicates a type strain. 


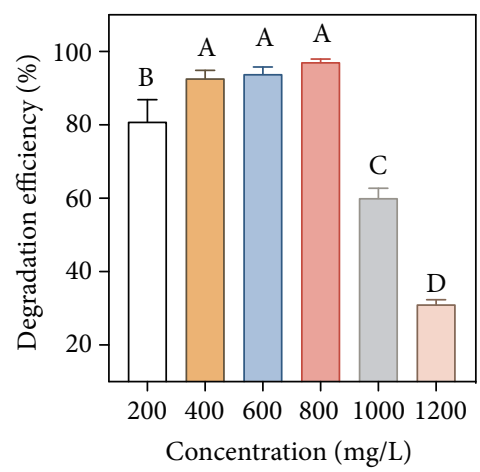

(a)

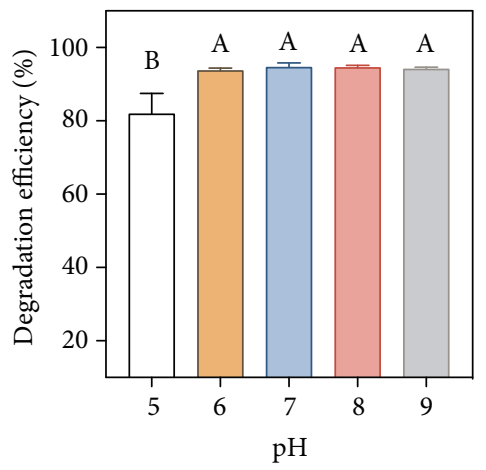

(b)

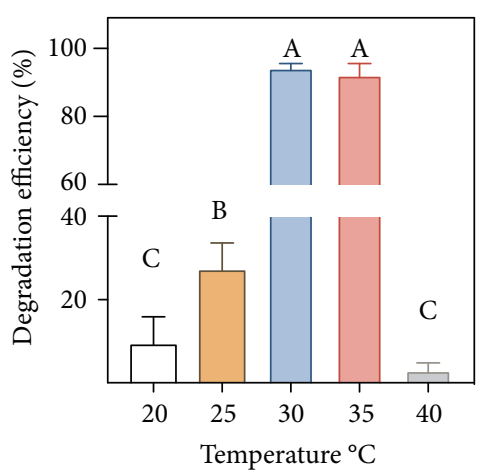

(c)

FIgURE 3: Effects of initial concentration (a), pH (b), and temperature (c) on the degradation of benzoic acid by strain SCB32. Values presented are means. Error bars represent the standard error of three replicates. Identical capital letters in each panel indicate no significant differences between treatments at $p<0.05$.

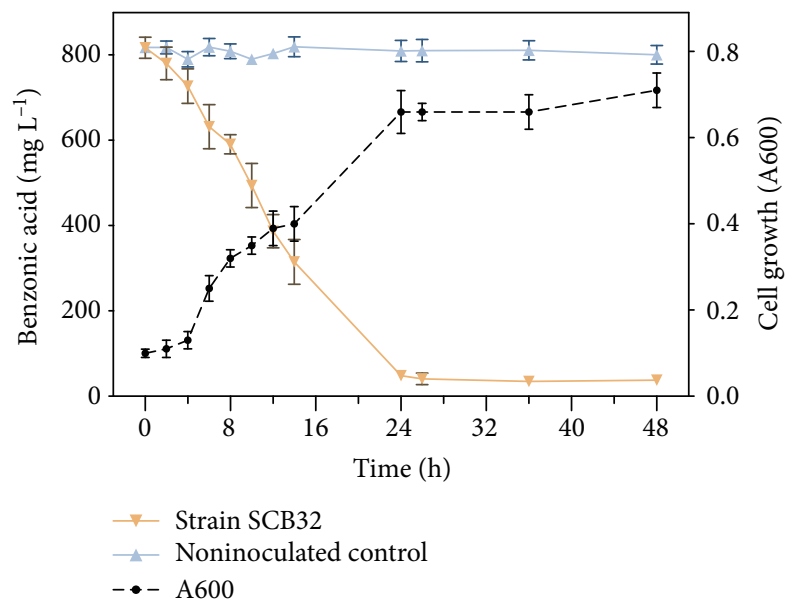

FIGURE 4: Cell growth and benzoic acid consumption of strain SCB32 in batch culture with initial concentration of $800 \mathrm{mg} \mathrm{L}^{-1}$ benzoic acid. Values presented are means. Error bars represent the standard error of three replicates.

degradation of benzoic acid was highest at $\mathrm{pH} 7.0$, with a mean degradation rate $>94.7 \%$. However, this degradation rate was not significantly different to those obtained at $\mathrm{pH} 6.0,8.0$, and $9.0(p<0.05)$. Temperature had a strong influence on the degradation rate. High $\left(40^{\circ} \mathrm{C}\right)$ and low $\left(20^{\circ} \mathrm{C}\right)$ temperatures were unfavorable for growth and degradation ability of strain SCB32, resulting in a degradation rate of less than $10.0 \%(p<0.05)$. The optimal degradation temperature was $30^{\circ} \mathrm{C}$ at $800 \mathrm{mg} \mathrm{L}^{-1}$ benzoic acid and resulted in a degradation rate $>94.3 \%$ (Figure $3(\mathrm{c})$ ).

Under the optimized conditions $\left(\mathrm{pH} 7.0,30^{\circ} \mathrm{C}\right.$, and $800 \mathrm{mg} \mathrm{L}^{-1}$ benzoic acid), a negligible acclimation phase was observed (Figure 4). Soon after, cell concentration increased exponentially and benzoic acid was degraded. At $12 \mathrm{~h}$, the benzoic acid had been degraded by more than $50 \%$, and by $24 \mathrm{~h}$, growth of the strain had slowed and the concentration of benzoic acid had dropped to an almost undetectable level.

3.3. Genome Sequencing and Annotation. The extracted total DNA concentration of strain SCB32 was $184 \mu \mathrm{g} \mu \mathrm{L}^{-1}$, and the
TABLE 1: Genome characteristics of strain SCB32.

\begin{tabular}{lc}
\hline Characteristic & Value \\
\hline Genome size (bp) & $6,311,241$ \\
GC content (\%) & 64.6 \\
No. of contigs & 4 \\
N50 contig length (bp) & $6,335,082$ \\
No. of genes & 5960 \\
Total size of all genes (bp) & $5,460,030$ \\
Average gene length (bp) & 960 \\
No. of tRNA & 68 \\
No. of rRNA & 19 \\
Genes assigned to COG & 4503 \\
Genes assigned to KEGG & 5414 \\
Genes assigned to GO & 3876 \\
Genes assigned to NR & 5493 \\
Genes assigned to Swiss-Prot & 2607 \\
\hline
\end{tabular}

OD260/280 value was 1.89 , which met the sequencing concentration and quality requirements. The genome sequence of strain SCB32 comprised 6,311,241 bp with a GC content of $64.6 \%$ (Table 1). Approximately 5960 coding genes and 71 repeated sequences were predicted. A graphical circular genome map of strain SCB32 is shown in Figure 5. Utilizing COG function assignment, 4503 coding genes could be classified into 25 COG function classes. Cellular components, molecular functions, and biological processes of strain SCB32 were also classified and revealed by genome functional annotation against the GO database. According to KEGG pathway mapping, $90.8 \%$ of the total number of coding genes could be assigned to 233 metabolic pathways (Figure 6). Among the predicted genes, 31 were associated with benzoic acid metabolism (Supplementary Table S3).

3.4. Identification of Benzoic Acid Metabolites. The benzoic acid degradation process was identified by GC-MS analysis. During the growth of the SCB32 cocultures with benzoate, three TMS derivatives of interest were detected during 

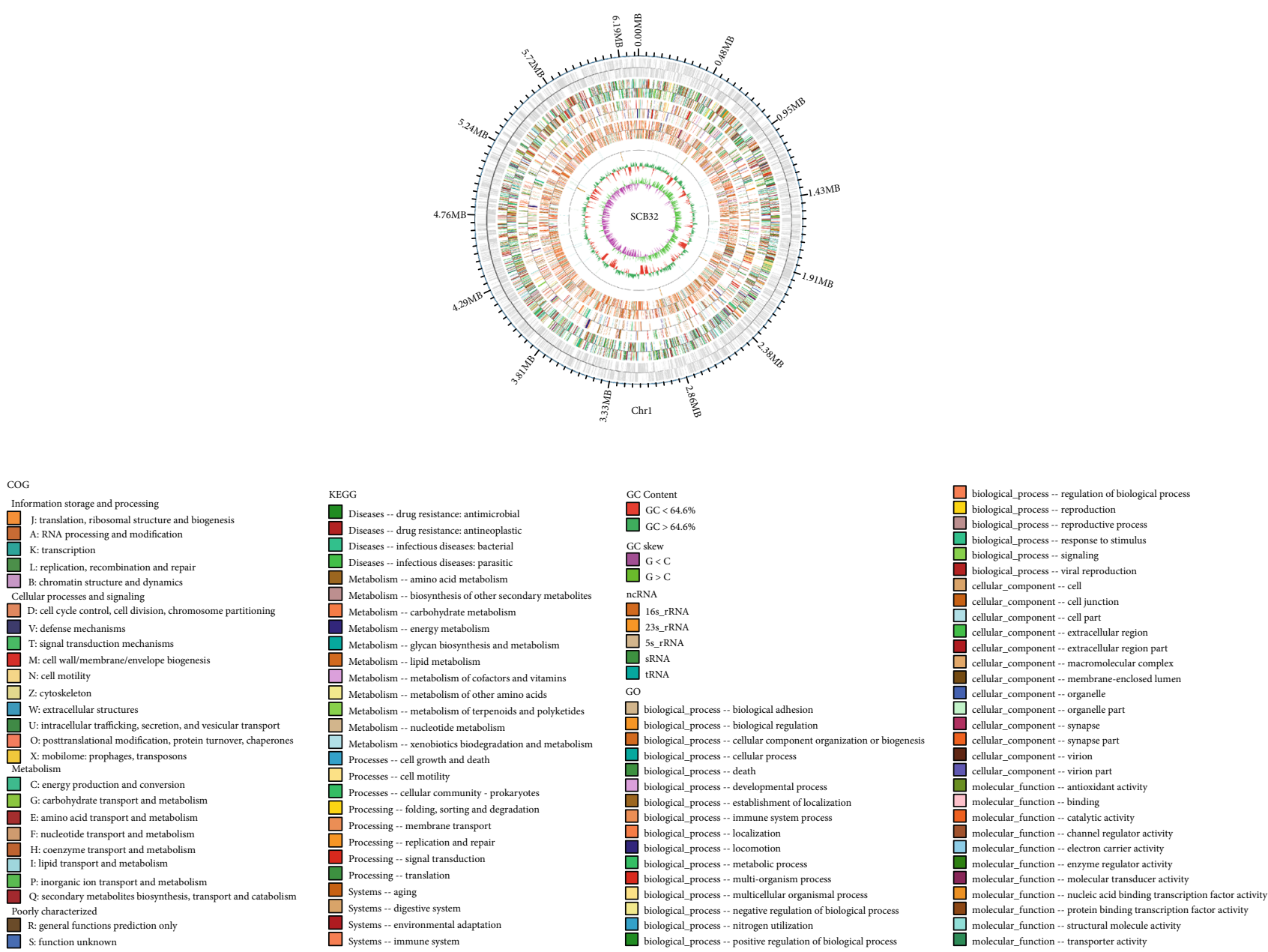

Figure 5: Circular chromosome of Pseudomonas sp. SCB32. Circular genome map generated by Circos and showing, from the outside inwards, the following: ring 1, genomic position; ring 2, genes assigned to COG categories; ring 3, genes assigned to KEGG database pathways; ring 4, genes assigned to GO database terms; ring 5, genes assigned to ncRNA; ring 6, GC content; and ring 7, GC skew.

different stages of the degradation process. The TMS derivative of each compound had the same retention time (RT) and mass spectral profile as the TMS derivative of the National Institute of Standards and Technology (NIST) database. During the $48 \mathrm{~h}$ of incubation, catechol-TMS (RT $7.76 \mathrm{~min}$ ) (Figure 7(b)) was detected first at $4 \mathrm{~h}$. The second compound, cis,cis-muconic acid-TMS (RT $11.27 \mathrm{~min}$ ) (Figure 7(c)), was detected at 4,6 , and $8 \mathrm{~h}$. The third compound, at $14 \mathrm{~h}$, was 3-oxoadipate-TMS (RT $9.76 \mathrm{~min}$ ) (Figure 7(d)). In sterile MSM cultures without inoculation of strain SCB32, benzoic acid changed little and no target metabolites were detected throughout the experiment. These results indicate that benzoic acid degradation by strain SCB32 occurs through the ortho pathway. Benzoic acid may be hydroxylated to form catechol, which is subsequently oxidized by ring fission to yield cis,cis-muconic acid, followed by muconolactone, and ultimately, downstream products enter the citrate cycle (TCA cycle).

3.5. Bioassay of the Benzoic Acid Degradation Products. The toxicity of benzoic acid and its degradation products were evaluated using lettuce seeds (Figures 8 and 9). Benzoic acid at $800 \mathrm{mg} \mathrm{L}^{-1}$ had a significant toxic effect on lettuce seeds, with a seed germination rate of $0 \%$. However, when the lettuce seeds were exposed to benzoic acid inoculated with strain SCB32, there was a significant detoxification effect. The degradation products of benzoic acid did not significantly inhibit seed germination rate, plant height, or fresh weight, which were $1.1 \%, 8.9 \%$, and $2.2 \%$ lower than the control (MSM alone), respectively. Interestingly, root length of the degradation product-treated group increased by $6.1 \%$ compared with the control.

\section{Discussion}

Allelochemicals, metabolites produced by living organisms that have a detrimental effect on other species when released into the environment, significantly inhibit plant growth [11, 37]. The use of microorganisms to degrade allelochemicals such as benzoic acid and for soil remediation has proven to be effective [38,39]. In this study, using an enrichment and domestication strategy, a benzoic acid-degrading bacterium, SCB32, was isolated and revealed to be a member of the genus Pseudomonas. The genus Pseudomonas, belonging to 

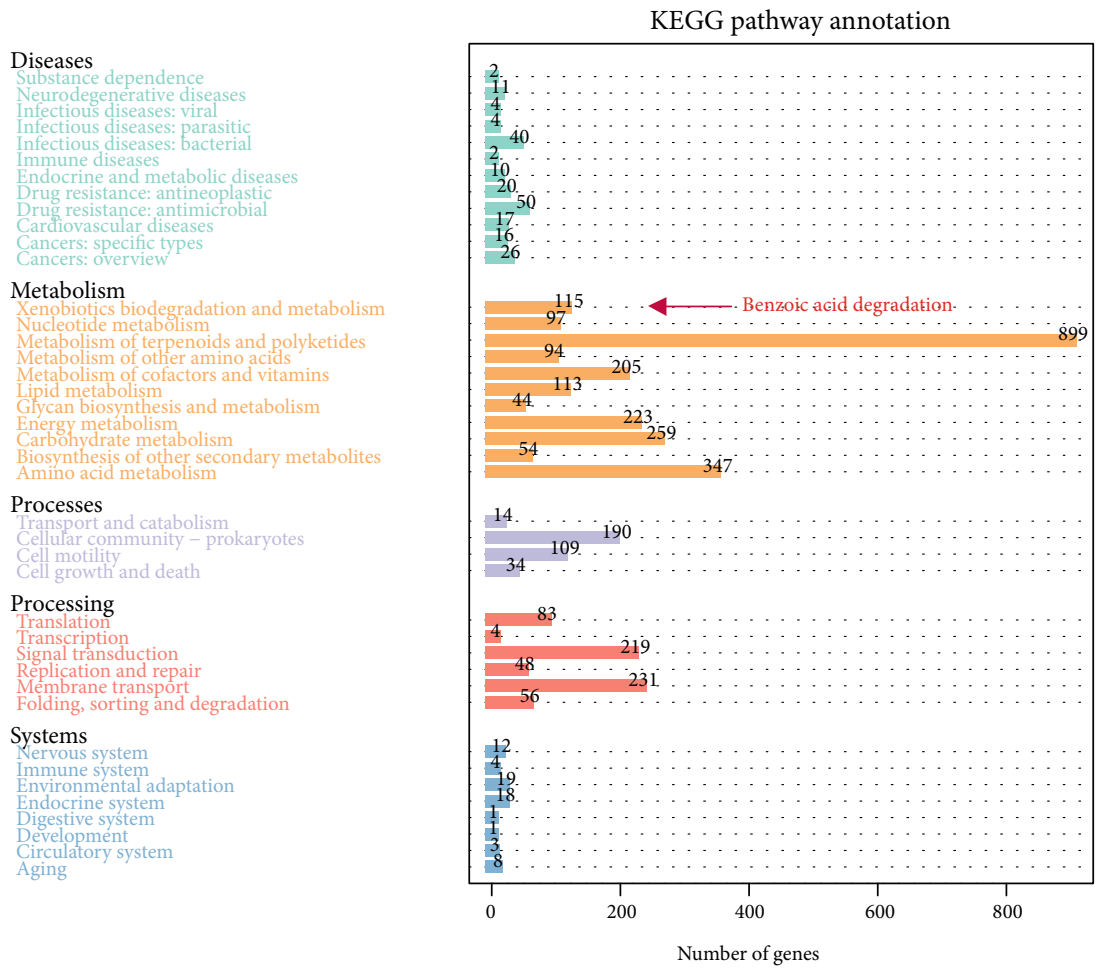

Figure 6: KEGG pathway annotation of genes of strain SCB32.

the family Pseudomonadaceae, is metabolically diverse and contains more than 190 species with validly published names [40]. The most closely related genus is Azomonas [41]. Modern bacterial taxonomy defines species by directly comparing whole genome sequences, and average nucleotide identity (ANI) has been widely recognized as a useful tool in this process [42]. The generally accepted cutoff value for the species boundary is approximately 95 96\% ANI [43, 44]. Using the TrueBac ID system [31], the ANI value between strain SCB32 and reference strains was far lower than the cutoff value (Supplementary Table S2). Unfortunately, combined with the identification results of physiological, biochemical, and molecular biology analyses, the specific classification of strain SCB32 cannot currently be determined. A more comprehensive identification of strain SCB32 will be conducted in future work.

Many bacterial species from the genus Pseudomonas have been reported to degrade benzoic acid or benzoate, but their degradation ability varies. In this study, when the concentration of benzoic acid was $800 \mathrm{mg} \mathrm{L}^{-1}$, strain SCB32 showed the best growth and the highest degradation rate $(>97 \%)$ in $24 \mathrm{~h}$. But above this concentration, an inhibitory effect was observed. This is because benzoic acid has a known antibacterial effect [5]. Previously reported biodegradable concentrations of benzoic acid were mostly below $500 \mathrm{mg} \mathrm{L}^{-1}$. For example, Pseudomonas sp. QTF5 can degrade $12.4 \%$ of benzoate at a concentration of $3 \mathrm{mg} \mathrm{mL}^{-1}$ within 3 days [45]. Pseudomonas putida ATCC 49451 degrades approximately $100 \%$ of benzoate at a concentration of $400 \mathrm{mg} \mathrm{L}^{-1}$ within $12 \mathrm{~h}$. For P. putida ATCC 49451, substrate inhibition was observed at benzoate concentrations higher than $500 \mathrm{mg} \mathrm{L}^{-1}$ [21]. Strain WH-B3 degrades more than $90 \%$ of benzoate at a concentration of $500 \mathrm{mg} \mathrm{L}^{-1}$ within $12 \mathrm{~h}$ [38]. Compared with them, stain SCB32 has a higher degradation capacity. In addition, strain SCB32 shows better $\mathrm{pH}$ adaptability for benzoic acid degradation. The benzoic acid degradation rate of strain SCB32 was reduced under specified temperature conditions, determined by the characteristics of the strain itself [29]. The concentrations of benzoic acid in the environment are far lower than the abovementioned tolerable value $\left(800 \mathrm{mg} \mathrm{L}^{-1}\right)$, indicating that strain SCB32 has great application potential. Although some environmental pollutants are degraded by microbes, they may produce toxic intermediates such as benzoic acid or catechol and cannot be further degraded $[46,47]$. Therefore, autotoxicity of the final degradation products of pollutants is of interest. Lettuce (Lactuca sativa L.) is generally accepted as an allelochemicalsensitive plant species, and benzoic acid has a significant toxic effect on it $[38,48]$. In this work, benzoic acid $\left(800 \mathrm{mg} \mathrm{L}^{-1}\right)$ was degraded by strain SCB32 within $48 \mathrm{~h}$, and the residues of degradation products were phenotypically nontoxic to lettuce seed germination. Strain SCB32 therefore has potential in the biodegradation of benzoic acid for agricultural applications.

The metabolic processes of most of the benzoic aciddegrading bacteria are based on presumed evidence, including the identification of metabolites and the detection of key enzyme activities [22, 49], and inferring metabolism is an imperfect task. In contrast, microbial genome analysis and gene annotation allowed us to directly display the various genes responsible for the biodegradation potential of benzoic acid [26], promoting investigation of environmental bioremediation by strain SCB32. In this study, the benzoic acid metabolic pathway based on genome annotation and 


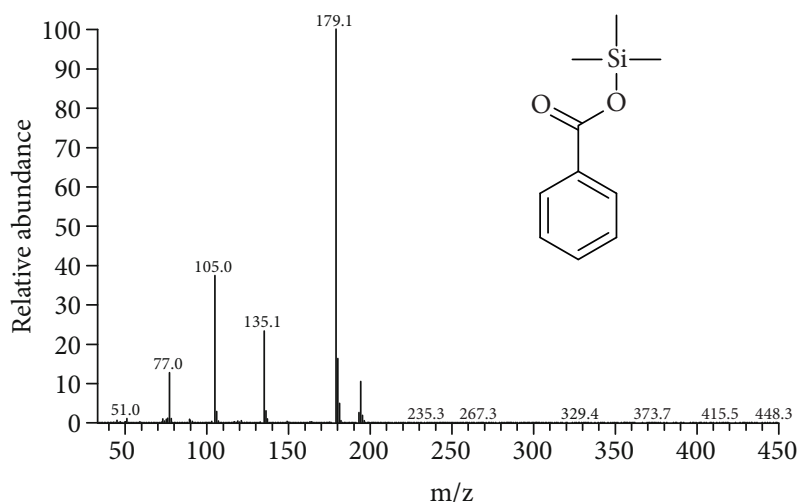

(a)

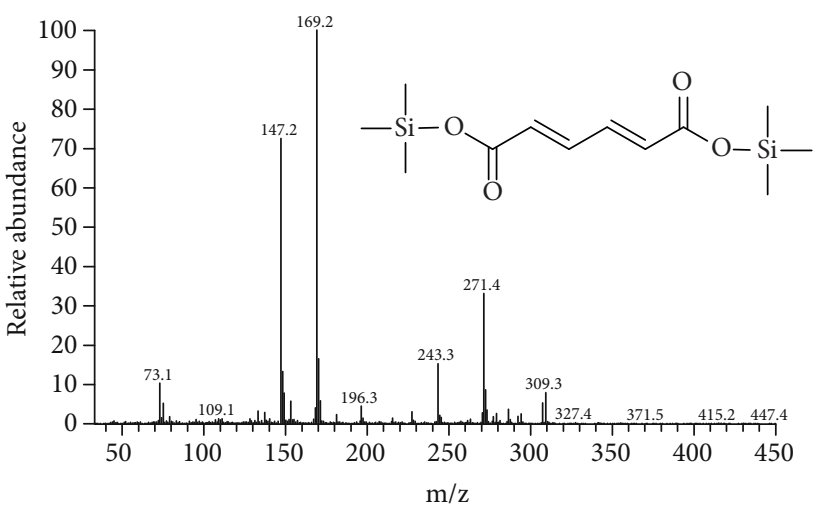

(c)

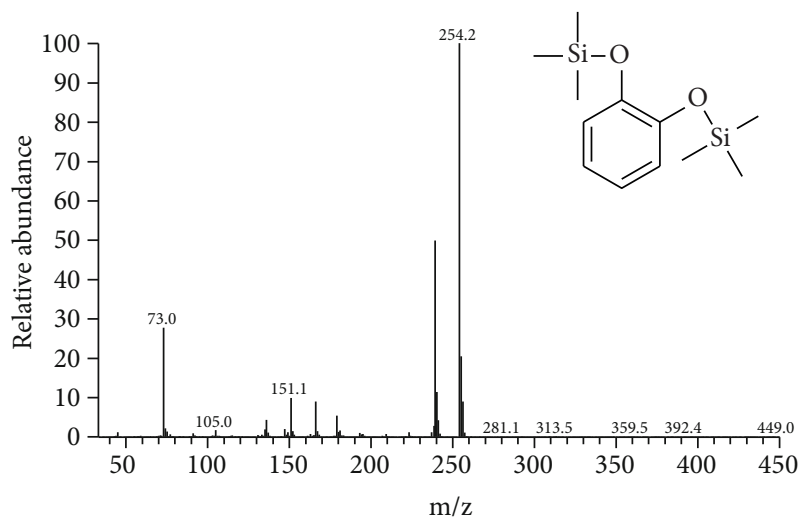

(b)

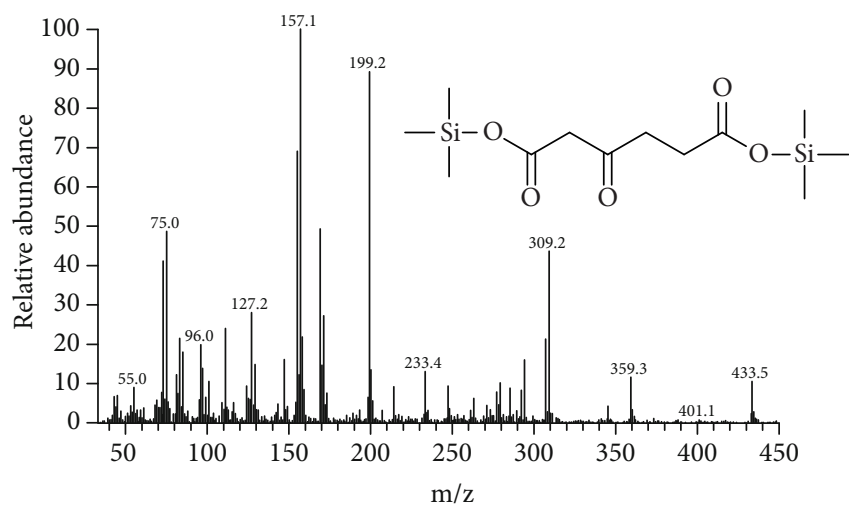

(d)

FIgURE 7: Mass spectra of TMS derivatives of metabolites detected in cultures of benzoic acid-grown strain SCB32. (a) Benzoic acid-TMS, (b) catechol-TMS, (c) cis,cis-muconic acid-TMS, (d) 3-oxoadipate-TMS.

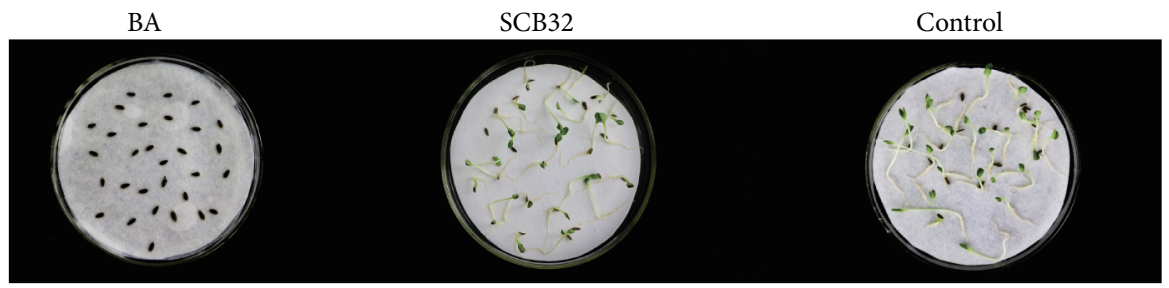

(a)

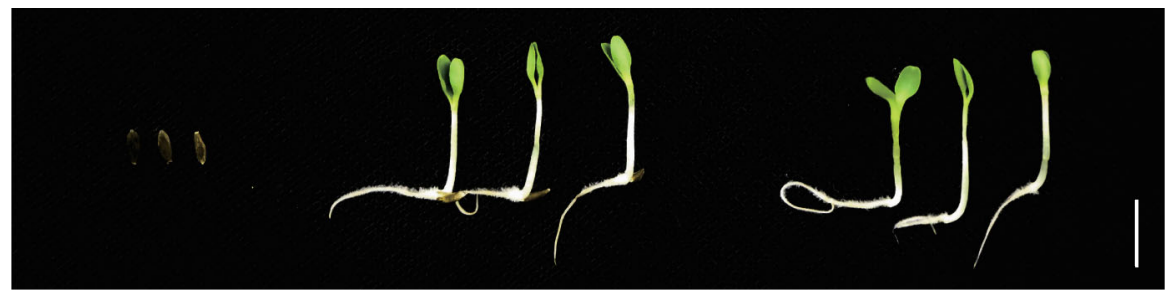

(b)

FIGURE 8: Lettuce seeds exposed to exogenous benzoic acid and its degradation metabolites. Lettuce seeds were treated with MSM (control), $800 \mathrm{mg} \mathrm{L}^{-1}$ benzoic acid (BA), or metabolites from BA degraded by SCB32. (a) Lettuce germination and (b) lettuce seeds after $7 \mathrm{~d}$ culture. Bar, $1 \mathrm{~cm}$.

MS was inferred (Figure 10). Initially, benzoic acid is converted into 1,2-dihydro-1,2-dihydroxybenzoic acid (DHB) by benzoate-1,2-dioxygenase (benA-xylX, benB-xylY, or ben $C-x y l Z$ gene), then transformed into catechol by the func- tion of the gene benD-xylL $[50,51]$. Catechol is an intermediate of many aromatic metabolites [52]. Next, catechol, transformed into cis,cis-muconate, undergoes ring cleavage by catechol-1,2-dioxygenase (catA gene) through the ortho 


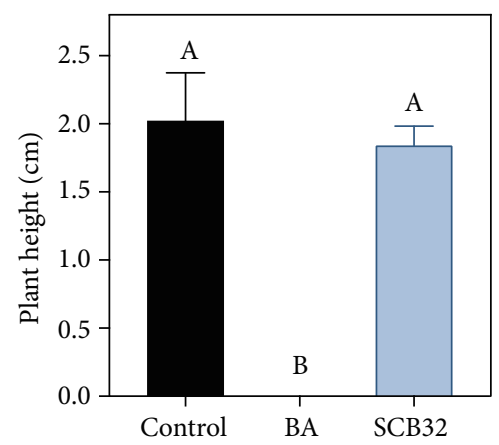

(a)

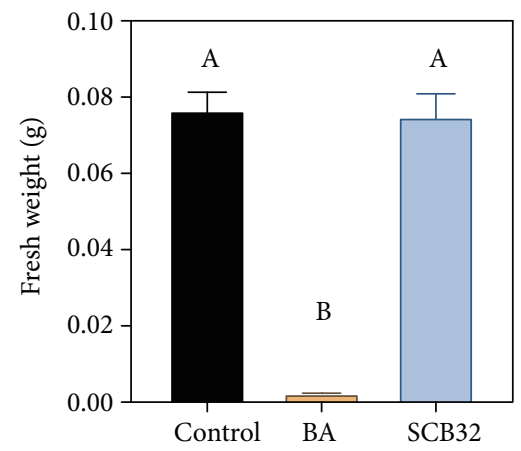

(c)

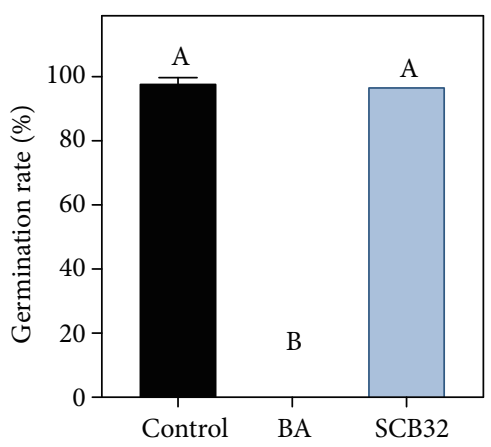

(b)

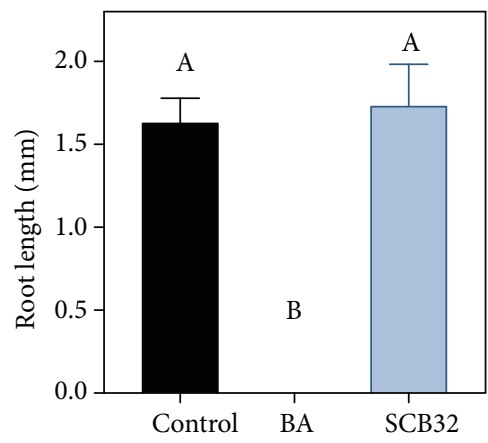

(d)

FIGURE 9: Bioassay on lettuce seeds of benzoic acid and its degradation metabolites produced by strain SCB32. Lettuce seeds were treated with MSM (control), $800 \mathrm{mg} \mathrm{L}^{-1}$ benzoic acid (BA), or metabolites from BA degraded by SCB32. Error bars represent the standard error of three replicates. Identical capital letters in each panel indicate no significant differences between treatments at $p<0.05$.

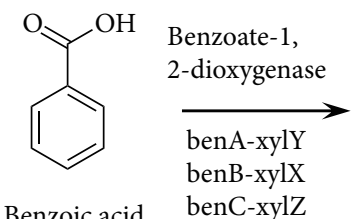

Benzoic acid<smiles>O=C(O)[C@]1(O)C=CC=C[C@H]1O</smiles>

DHB

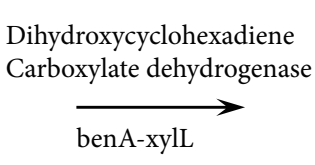

benA-xylL<smiles>Oc1ccccc1O</smiles>

Catechol

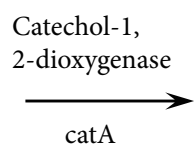

catA<smiles>O=C(O)/C=C\C(=O)O</smiles>

cis,cis-Muconate

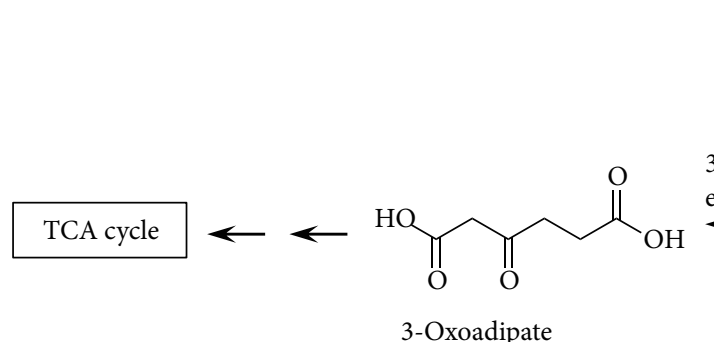

Muconate cycloisomerase

catB

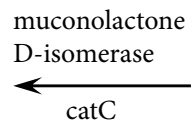

catC<smiles>O=C(O)C[C@H]1C=CC(=O)O1</smiles>

(+)-Muconolactone

FIGURE 10: Proposed pathway for benzoic acid metabolism by strain SCB32. Asterisks indicate compounds detected as TMS derivatives in SCB32 coculture extracts.

pathway [53]. Subsequently, the metabolites of each process undergo a series of transformations under functional genes such as $c a t B$, cat $C$, and $p c a D$ and finally degrade to tricarboxylic acid (TCA) cycle [54, 55]. Moreover, using GC-MS analysis, catechol, cis,cis-muconic acid, and 3-oxoadipate were identified. This is consistent with the pathway based on genome prediction. Some intermediate metabolites were not detected, because they transformed either too fast or at a low level. For example, 1,2-dihydro-1,2-dihydroxybenzoic acid is a very unstable intermediate and is usually consumed rapidly [56]. Comprehensive understanding of the degradation mechanism may help with continued enhancement of the process of bioremediation.

\section{Conclusions}

Data obtained in this study indicate that strain SCB32 could degrade a model allelochemical benzoic acid as the sole carbon source for growth and that it could effectively degrade benzoic acid in MSM. The biodegradation pathway 
of benzoic acid, the ortho pathway, is proposed for strain SCB32 based on combined data from genome annotation and mass spectrometry. Furthermore, the degradation products of benzoic acid by strain SCB32 had no obvious toxic effect on lettuce germination. Our results provide the groundwork for further elucidation of the genetic basis of benzoic acid degradation in strain SCB32. These results indicate a possible application of strain SCB32 in the bioremediation of benzoic acid contamination in agricultural environments.

\section{Data Availability}

The 16S rRNA gene sequence and genome sequence of strain SCB32 were submitted to the NCBI GenBank database under accession numbers MN559810 and CP045118, respectively.

\section{Conflicts of Interest}

The authors declare that there is no conflict of interest regarding the publication of this paper.

\section{Authors' Contributions}

Wei Xiang, Liangbo Li, and Rongshao Huang conceived and designed the study. Wei Xiang and Xiaolan Wei performed the experiments. Wei Xiang performed bacterial identifications and analyzed the genomic data. Liangbo Li, Rongshao Huang, and Hui Tang contributed to materials and analysis tools. All authors read and approved the final manuscript.

\section{Acknowledgments}

This research was funded by the National Natural Science Foundation of China (Grant No. 81860669) and the Science and Technology Major Project of Guangxi (Grant Nos. AA17204056 and AA18118015).

\section{Supplementary Materials}

Table S1: physiological and biochemical characteristic by VITEK GN. Table S2: average nucleotide identity (ANI). Table S3: potential benzoic acid degradation genes found in the isolate SCB322 genome annotated by the KEGG database. (Supplementary Materials)

\section{References}

[1] H. I. Abdel-Shafy and M. S. M. Mansour, "A review on polycyclic aromatic hydrocarbons: source, environmental impact, effect on human health and remediation," Egyptian Journal of Petroleum, vol. 25, no. 1, pp. 107-123, 2016.

[2] Z.-Y. Zhang, L.-P. Pan, and H.-H. Li, "Isolation, identification and characterization of soil microbes which degrade phenolic allelochemicals," Journal of Applied Microbiology, vol. 108, no. 5, pp. 1839-1849, 2010.

[3] N. Perchat, P.-L. Saaidi, E. Darii et al., "Elucidation of the trigonelline degradation pathway reveals previously undescribed enzymes and metabolites," Proceedings of the National Academy of Sciences, vol. 115, no. 19, pp. E4358-E4367, 2018.
[4] L. Ren, Y. Jia, R. Zhang et al., "Insight into metabolic versatility of an aromatic compounds-degrading Arthrobacter sp. YCRL1," Frontiers in Microbiology, vol. 9, 2018.

[5] A. del Olmo, J. Calzada, and M. Nuñez, "Benzoic acid and its derivatives as naturally occurring compounds in foods and as additives: uses, exposure, and controversy," Critical Reviews in Food Science and Nutrition, vol. 57, no. 14, pp. 3084-3103, 2015.

[6] H. P. Singh, D. R. Batish, and R. K. Kohli, "Autotoxicity: concept, organisms, and ecological significance," Critical Reviews in Plant Sciences, vol. 18, no. 6, pp. 757-772, 1999.

[7] H. Ozpinar, S. Dag, and E. Yigit, "Alleophatic effects of benzoic acid, salicylic acid and leaf extract of Persica vulgaris Mill. (Rosaceae)," South African Journal of Botany, vol. 108, pp. 102-109, 2017.

[8] W. Zhu, J. Liu, J. Ye, and G. Li, "Effects of phytotoxic extracts from peach root bark and benzoic acid on peach seedlings growth, photosynthesis, antioxidance and ultrastructure properties," Scientia Horticulturae, vol. 215, pp. 49-58, 2017.

[9] W. Zhang, L.-Y. Lu, L.-Y. Hu et al., "Evidence for the involvement of auxin, ethylene and ROS signaling during primary root inhibition of arabidopsis by the allelochemical benzoic acid," Plant and Cell Physiology, vol. 59, no. 9, pp. 1889-1904, 2018.

[10] Y. Liu, X. Li, K. Cai, L. Cai, N. Lu, and J. Shi, "Identification of benzoic acid and 3-phenylpropanoic acid in tobacco root exudates and their role in the growth of rhizosphere microorganisms," Applied Soil Ecology, vol. 93, pp. 78-87, 2015.

[11] J. Liu, X. Li, Z. Jia, T. Zhang, and X. Wang, "Effect of benzoic acid on soil microbial communities associated with soilborne peanut diseases," Applied Soil Ecology, vol. 110, pp. 34-42, 2017.

[12] Y. Li, B. Wang, Y. Chang et al., "Reductive soil disinfestation effectively alleviates the replant failure of Sanqi ginseng through allelochemical degradation and pathogen suppression," Applied Microbiology and Biotechnology., vol. 103, no. 8, pp. 3581-3595, 2019.

[13] M. Schulz, M. Siebers, and N. Anders, "Exploring plants strategies for allelochemical detoxification," in Advances in Plant Ecophysiology Techniques, A. M. Sánchez-Moreiras and M. J. Reigosa, Eds., pp. 379-399, Springer International Publishing, Cham, 2018.

[14] Z. Wu, Z. Hao, Y. Zeng, L. Guo, L. Huang, and B. Chen, "Molecular characterization of microbial communities in the rhizosphere soils and roots of diseased and healthy Panax notoginseng," Antonie Van Leeuwenhoek, vol. 108, no. 5, pp. 1059-1074, 2015.

[15] M. Yang, X. Zhang, Y. Xu et al., "Autotoxic ginsenosides in the rhizosphere contribute to the replant failure of Panax notoginseng," PLOS ONE, vol. 10, no. 2, article e0118555, 2015.

[16] W. Xiang, Autotoxicity in Panax notoginseng of root exudates and their allelochemicals, Master's Thesis, Guangxi University, Nanning, 2016, [in Chinese].

[17] M. S. Elshahed, V. K. Bhupathiraju, N. Q. Wofford, M. A. Nanny, and M. J. McInerney, "Metabolism of benzoate, cyclohex-1-ene carboxylate, and cyclohexane carboxylate by "Syntrophus aciditrophicus" strain SB in syntrophic association with H2-using microorganisms," Applied and Environmental Microbiology, vol. 67, no. 4, pp. 1728-1738, 2001.

[18] S. Ghosh, A. Qureshi, and H. J. Purohit, "Enhanced expression of catechol 1,2 dioxygenase gene in biofilm forming Pseudomonas mendocina EGD-AQ5 under increasing 
benzoate stress," International Biodeterioration \& Biodegradation, vol. 118, pp. 57-65, 2017.

[19] L. Ma, S. Chen, J. Yuan, P. Yang, Y. Liu, and K. Stewart, "Rapid biodegradation of atrazine by Ensifer sp. strain and its degradation genes," International Biodeterioration \& Biodegradation, vol. 116, pp. 133-140, 2017.

[20] P. A. Williams and J. R. Sayers, "The evolution of pathways for aromatic hydrocarbon oxidation inPseudomonas," Biodegradation, vol. 5, no. 3-4, pp. 195-217, 1994.

[21] K.-C. Loh and S.-S. Chua, "Ortho pathway of benzoate degradation in Pseudomonas putida: induction of meta pathway at high substrate concentrations," Enzyme and Microbial Technology, vol. 30, no. 5, pp. 620-626, 2002.

[22] B. Cao, A. Geng, and K.-C. Loh, "Induction of ortho- and meta-cleavage pathways in Pseudomonas in biodegradation of high benzoate concentration: MS identification of catabolic enzymes," Applied Microbiology and Biotechnology, vol. 81, no. 1, pp. 99-107, 2008.

[23] V. J. Denef, J. Park, T. V. Tsoi et al., "Biphenyl and benzoate metabolism in a genomic context: outlining genome-wide metabolic networks in Burkholderia xenovorans LB400," Applied Microbiology and Biotechnology, vol. 70, no. 8, pp. 4961-4970, 2004.

[24] R. Fleischmann, M. Adams, O. White et al., "Whole-genome random sequencing and assembly of Haemophilus influenzae Rd," Science, vol. 269, no. 5223, pp. 496-512, 1995.

[25] E. W. Sayers, M. Cavanaugh, K. Clark, J. Ostell, K. D. Pruitt, and I. Karsch-Mizrachi, "GenBank," Nucleic Acids Research, vol. 47, no. D1, pp. D94-D99, 2018.

[26] H. P. J. Buermans and J. T. den Dunnen, "Next generation sequencing technology: advances and applications," Biochimica et Biophysica Acta (BBA) - Molecular Basis of Disease, vol. 1842, no. 10, pp. 1932-1941, 2014.

[27] H. Shao, M. Chen, X. Fei et al., "Complete genome sequence and characterization of a polyethylene biodegradation strain, Streptomyces albogriseolus LBX-2," Microorganisms, vol. 7, no. 10 , p. $379,2019$.

[28] M. Mahboubifar, Z. Sobhani, G. Dehghanzadeh, and K. Javidnia, "A comparison between UV spectrophotometer and high-performance liquid chromatography method for the analysis of sodium benzoate and potassium Sorbate in food products," Food Analytical Methods, vol. 4, no. 2, pp. 150-154, 2011.

[29] Y.-N. Wang, W.-H. He, H. He et al., "Pseudomonas nitritireducens sp. nov., a nitrite reduction bacterium isolated from wheat soil," Archives of Microbiology, vol. 194, no. 10, pp. 809-813, 2012.

[30] S.-H. Yoon, S.-M. Ha, S. Kwon et al., "Introducing EzBioCloud: a taxonomically united database of $16 \mathrm{~S}$ rRNA gene sequences and whole-genome assemblies," International Journal of Systematic and Evolutionary Microbiology., vol. 67, no. 5, pp. 1613-1617, 2017.

[31] S. M. Ha, C. K. Kim, J. Roh et al., "Application of the whole genome-based bacterial identification system, TrueBac ID, using clinical isolates that were not identified with three matrix-assisted laser desorption/ionization time-of-flight mass spectrometry (MALDI-TOF MS) systems," Annals of Laboratory Medicine, vol. 39, no. 6, pp. 530-536, 2019.

[32] M. Ashburner, C. A. Ball, J. A. Blake et al., "Gene ontology: tool for the unification of biology," Nature Genetics, vol. 25, no. 1, pp. 25-29, 2000.
[33] M. Kanehisa, S. Goto, S. Kawashima, Y. Okuno, and M. Hattori, "The KEGG resource for deciphering the genome," Nucleic Acids Research, vol. 32, no. 90001, supplement 1, pp. 277D-2280, 2004.

[34] M. Y. Galperin, K. S. Makarova, Y. I. Wolf, and E. V. Koonin, "Expanded microbial genome coverage and improved protein family annotation in the COG database," Nucleic Acids Research, vol. 43, no. D1, pp. D261-D269, 2015.

[35] W. Li, L. Jaroszewski, and A. Godzik, “Tolerating some redundancy significantly speeds up clustering of large protein databases," Bioinformatics, vol. 18, no. 1, pp. 77-82, 2002.

[36] A. Bairoch and R. Apweiler, "The SWISS-PROT protein sequence database and its supplement TrEMBL in 2000," Nucleic Acids research, vol. 28, no. 1, pp. 45-48, 2000.

[37] M. Yang, Y. Chuan, C. Guo et al., "Panax notoginseng root cell death caused by the autotoxic ginsenoside $\operatorname{Rg} 1$ is due to overaccumulation of ROS, as revealed by transcriptomic and cellular approaches," Frontiers in Plant Science, vol. 9, 2018.

[38] H. He, W. Zhu, I. Noor, J. Liu, and G. Li, "Pseudomonas putida WH-B3 degrades benzoic acid and alleviates its autotoxicity to peach (Prunus persica L. batsch) seedlings grown in replanted soil," Scientia Horticulturae, vol. 255, pp. 183-192, 2019.

[39] L. Ren, Y. Jia, N. Ruth et al., "Biodegradation of phthalic acid esters by a newly isolated Mycobacterium sp. YC-RL4 and the bioprocess with environmental samples," Environmental Science and Pollution Research, vol. 23, no. 16, pp. 1660916619, 2016.

[40] A. Peix, M.-H. Ramírez-Bahena, and E. Velázquez, "The current status on the taxonomy of Pseudomonas revisited: an update," Infection, Genetics and Evolution, vol. 57, pp. 106$116,2018$.

[41] A. I. Özen and D. W. Ussery, "Defining the Pseudomonas genus: where do we draw the line with Azotobacter?," Microbial Ecology, vol. 63, no. 2, pp. 239-248, 2012.

[42] J. Chun and F. A. Rainey, "Integrating genomics into the taxonomy and systematics of the Bacteria and Archaea," International Journal of Systematic and Evolutionary Microbiology, vol. 64, Part 2, pp. 316-324, 2014.

[43] I. Lee, Y. Ouk Kim, S.-C. Park, and J. Chun, “OrthoANI: an improved algorithm and software for calculating average nucleotide identity," International Journal of Systematic and Evolutionary Microbiology, vol. 66, no. 2, pp. 1100-1103, 2016.

[44] M. Richter and R. Rosselló-Móra, "Shifting the genomic gold standard for the prokaryotic species definition," PNAS, vol. 106, no. 45, pp. 19126-19131, 2009.

[45] Y. Li, Y. Ren, and N. Jiang, "Analysis of draft genome sequence of Pseudomonas sp. QTF5 reveals its benzoic acid degradation ability and heavy metal tolerance," BioMed Research International, vol. 2017, 7 pages, 2017.

[46] D.-W. Gao and Z.-D. Wen, "Phthalate esters in the environment: a critical review of their occurrence, biodegradation, and removal during wastewater treatment processes," Science of The Total Environment., vol. 541, pp. 986-1001, 2016.

[47] L. P. Wackett, "Pseudomonas putida-a versatile biocatalyst," Nature Biotechnology, vol. 21, no. 2, pp. 136-138, 2003.

[48] J. G. Lee, B. Y. Lee, and H. J. Lee, “Accumulation of phytotoxic organic acids in reused nutrient solution during hydroponic cultivation of lettuce (Lactuca sativa L.)," Scientia Horticulturae, vol. 110, no. 2, pp. 119-128, 2006.

[49] C. S. I. Oie, C. E. Albaugh, and B. M. Peyton, "Benzoate and salicylate degradation by Halomonas campisalis, an alkaliphilic 
and moderately halophilic microorganism," Water Research, vol. 41, no. 6, pp. 1235-1242, 2007.

[50] S. Harayama, M. Rekik, A. Bairoch, E. L. Neidle, and L. N. Ornston, "Potential DNA slippage structures acquired during evolutionary divergence of Acinetobacter calcoaceticus chromosomal benABC and Pseudomonas putida TOL pWW0 plasmid xylXYZ, genes encoding benzoate dioxygenases," Journal of Bacteriology, vol. 173, no. 23, pp. 7540-7548, 1991.

[51] E. Neidle, C. Hartnett, L. N. Ornston, A. Bairoch, M. Rekik, and S. Harayama, "Cis-diol dehydrogenases encoded by the TOL pWW0 plasmid xylL gene and the Acinetobacter calcoaceticus chromosomal benD gene are members of the shortchain alcohol dehydrogenase superfamily," European Journal of Biochemistry, vol. 204, no. 1, pp. 113-120, 1992.

[52] I. A. Suvorova and M. S. Gelfand, "Comparative genomic analysis of the regulation of aromatic metabolism in Betaproteobacteria," Frontiers in Microbiology, vol. 10, 2019.

[53] E. L. Neidle, C. Hartnett, S. Bonitz, and L. N. Ornston, "DNA sequence of the Acinetobacter calcoaceticus catechol 1,2-dioxygenase I structural gene catA: evidence for evolutionary divergence of intradiol dioxygenases by acquisition of DNA sequence repetitions," Journal of Bacteriology, vol. 170, no. 10, pp. 4874-4880, 1988.

[54] M. Carmona, M. T. Zamarro, B. Blázquez et al., "Anaerobic catabolism of aromatic compounds: a genetic and genomic view," Microbiology and Molecular Biology Reviews, vol. 73, no. 1, pp. 71-133, 2009.

[55] J. E. Houghton, T. M. Brown, A. J. Appel, E. J. Hughes, and L. N. Ornston, "Discontinuities in the evolution of Pseudomonas putida cat genes," Journal of Bacteriology, vol. 177, no. 2, pp. 401-412, 1995.

[56] H. J. Chae and Y. J. Yoo, "Mathematical modelling and simulation of catechol production from benzoate using resting cells of Pseudomonas putida," Process Biochemistry, vol. 32, no. 5, pp. 423-432, 1997. 